\title{
Review
}

https://doi.org/10.1631/jzus.B2000319

\section{ADP-ribosylhydrolases: from DNA damage repair to COVID-19}

\author{
Lily YU ${ }^{1}$, Xiuhua LIU $^{2 \varpi}$, Xiaochun YU ${ }^{3 凶}$ \\ ${ }^{I}$ Westridge School, Pasadena, California 91105, USA \\ ${ }^{2}$ Institute of Life Science and Green Development, College of Life Science, Hebei University, Baoding 071002, China \\ ${ }^{3}$ School of Life Sciences, Westlake University, Hangzhou 310024, China
}

\begin{abstract}
Adenosine diphosphate (ADP)-ribosylation is a unique post-translational modification that regulates many biological processes, such as DNA damage repair. During DNA repair, ADP-ribosylation needs to be reversed by ADP-ribosylhydrolases. A group of ADP-ribosylhydrolases have a catalytic domain, namely the macrodomain, which is conserved in evolution from prokaryotes to humans. Not all macrodomains remove ADP-ribosylation. One set of macrodomains loses enzymatic activity and only binds to ADP-ribose (ADPR). Here, we summarize the biological functions of these macrodomains in DNA damage repair and compare the structure of enzymatically active and inactive macrodomains. Moreover, small molecular inhibitors have been developed that target macrodomains to suppress DNA damage repair and tumor growth. Macrodomain proteins are also expressed in pathogens, such as severe acute respiratory syndrome coronavirus 2 (SARS-CoV-2). However, these domains may not be directly involved in DNA damage repair in the hosts or pathogens. Instead, they play key roles in pathogen replication. Thus, by targeting macrodomains it may be possible to treat pathogen-induced diseases, such as coronavirus disease 2019 (COVID-19).
\end{abstract}

Key words: DNA damage repair; Severe acute respiratory syndrome coronavirus 2 (SARS-CoV-2); Adenosine diphosphate (ADP)-ribosylation; Macrodomain; ADP-ribosylhydrolase; deADP-ribosylation

\section{Human ADP-ribosylhydrolases}

In response to DNA damage, adenosine diphosphate (ADP)-ribosylation, including both poly (ADPribosyl)ation (aka PARylation) and mono (ADPribosyl)ation (aka MARylation), is quickly catalyzed by the "writers" poly(ADP-ribose) polymerases (PARPs) at DNA lesions and recognized by ADP ribose (ADPR)binding proteins ( $\mathrm{Li}$ and $\mathrm{Yu}, 2015$ ). Since many DNA damage repair proteins contain the ADPR-binding motifs, these "readers" facilitate DNA damage repair. Thus, ADPribosylation is one of the earliest signals at DNA lesions and mediates the first wave of DNA damage response.

In addition to the "writers" and "readers," the "erasers" ADP-ribosylhydrolases also play important

\footnotetext{
$凶$ Xiaochun YU, yuxiaochun@westlake.edu.cn

Xiuhua LIU, liuxiuhua_2004@163.com

(DD Xiuhua LIU, https://orcid.org/0000-0001-9734-8772
}

Received May 8, 2020; Revision accepted Aug. 10, 2020; Crosschecked Dec. 10, 2020

(c) Zhejiang University Press 2021 roles in DNA damage repair. Since ADP-ribosylation mediates the recruitment of DNA damage repair factors to DNA lesions, ADP-ribosylhydrolases need to reverse ADP-ribosylation to unload DNA damage repair factors from ADP-ribosylation sites (Liu et al., 2017; Kassab and Yu, 2019). If the removal of ADPribosylation is suppressed, DNA damage repair factors will be trapped at the sites of ADP-ribosylation because of high affinity between those repair factors and ADPR (Grundy et al., 2013; Krietsch et al., 2013). Thus, one of the biological functions of ADP-ribosylhydrolases is to release DNA damage repair factors from ADP-ribosylation for the next step of DNA damage repair. The removal of ADPribosylation (deADP-ribosylation) is not an antagonistic step of ADP-ribosylation during DNA damage repair. Instead, it is a sequential step in DNA damage repair.

Different ADP-ribosylhydrolases can reverse different forms of ADP-ribosylation (Fig. 1). Poly(ADP-ribose) glycohydrolase (PARG) is the major enzyme to remove PARylation and accounts for 
more than $90 \%$ of dePARylation during DNA damage repair (Min et al., 2010; Shirai et al., 2013). It is a polypeptide with 976 amino acids localizing in several cellular compartments including the nucleus, cytoplasm, and mitochondria. PARylation is a polymeric form of ADP-ribosylation, in which ADPR residues are covalently linked by a 1'-2' glycosidic bond (Talhaoui et al., 2016; Rack et al., 2020). The enzymatic domain of PARG is a macrodomain at its C-terminus, which has both endo- and exo-glycosidase activity for the cleavage of the 1'-2' glycosidic bond between two ADPR residues in poly(ADP-ribose) (PAR) chains. However, it cannot cut the ester bond between the first ADPR and amino acid acceptors, and leaves a single ADPR attached to the substrate (Lambrecht et al., 2015). Thus, PARG trims PARylation into MARylation during DNA damage repair (Fig. 1). The enzymatic activity of this macrodomain is very potent, as it can completely remove PARylation within 10-30 min following DNA damage. Although the structure of PARG's macrodomain has been solved, its detailed activation mechanism remains elusive (Feijs et al., 2013; Jankevicius et al., 2013; Rosenthal et al., 2013). While the PARG macrodomain itself clearly recognizes PAR (Barkauskaite et al., 2013; Lambrecht et al., 2015), which may directly trigger dePARylation, there should be other mechanisms for specific PARG activation through sensing extended PAR areas and/or protein interactors. For example, in addition to the Cterminal macrodomain, PARG has an $\mathrm{N}$-terminal regulator domain for catching the substrates or targeting PARG to the sites of DNA damage. The binding between the $\mathrm{N}$-terminal regulator domain and its functional partners, such as proliferating cell nuclear antigen (PCNA), may induce intermolecular changes for the activation of the macrodomain of PARG (Kaufmann et al., 2017). In addition to the role of macrodomains in protein deADP-ribosylation, some catalytic macrodomains are known to act on ADPR 1"-phosphate (Shull et al., 2005), ADP-ribosylated nucleic acids (Munnur and Ahel, 2017), and $O$-acetylADPR (Chen et al., 2011).

Accumulating evidence shows that PARG participates in DNA damage repair. Following DNA damageinduced PARylation, PARG quickly relocates the sites of DNA damage (Patel et al., 2005; Fisher et al., 2007). Cells lacking PARG have severe defects in the repair of DNA double-strand breaks (DSBs) and

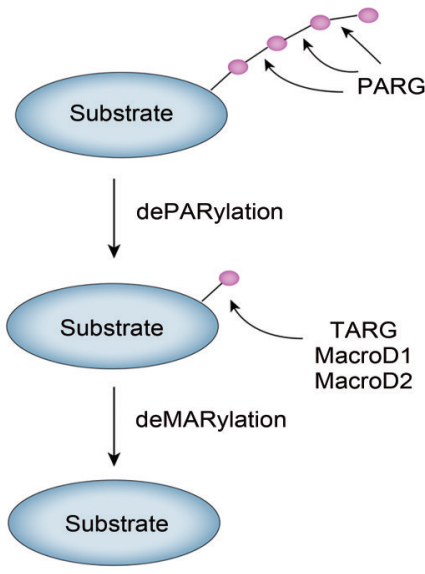

Fig. 1 Schematic of (dePARylation) and (deMARylation). The removal of adenosine diphosphate (ADP)-ribosylation, poly(ADP-ribosyl)ation (dePARylation), and mono(ADPribosyl)ation (deMARylation) mediated by macrodomaincontaining ADP-ribosylhydrolases including poly(ADPribose) glycohydrolase (PARG), terminal ADP-ribose protein glycohydrolase 1 (TARG1), MacroD1, and MacroD2.

single-strand breaks (SSBs) (Amé et al., 2009; Gogola et al., 2019). Because dePARylation is a downstream step of PARylation during DNA damage repair, suppression of the enzymatic activity of PARG's marcodomain will also impair DNA damage repair. If tumor cells have inherited mutations, such as breast cancer susceptibility gene 1 (BRCA1) or BRCA2 mutations, to partially abolish DNA damage repair, targeting the macrodomain, similar to targeting PARPs, will induce tumor cell apoptosis via synthetic lethality (Powell and Kachnic, 2003; Bryant et al., 2005; Audeh et al., 2010; Michels et al., 2014; Garufi et al., 2020). Based on this hypothesis, several small molecule inhibitors for targeting the macrodomain of PARG have been developed recently. These inhibitor treatments prolong the half-life of PARylation at the sites of DNA damage, trap DNA damaging factors, suppress DNA damage repair, and induce apoptosis of tumor cells with DNA damage repair defects (Fauzee et al., 2010; Gogola et al., 2019; Slade, 2020). Thus, it is possible that like PARP inhibitors, PARG inhibitors could in future be used for personalized cancer treatment. In addition, a sub-class of preclinical ovarian cancer models is sensitive to PARG inhibitors owing to potential DNA replication vulnerabilities (Pillay et al., 2019).

In addition to PARG, several other ADPribosylhydrolases, including terminal ADPR protein glycohydrolase 1 (TARG1), MacroD1, and MacroD2, 
also have a macrodomain (Rosenthal et al., 2013; Sharifi et al., 2013; Kassab et al., 2020). Compared to PARG, these are much smaller proteins. They have a macrodomain, but lack any regulatory domain, suggesting that the activation of these enzymes may be directly induced via the recognition of ADP-ribosylation by the macrodomain. Moreover, unlike the macrodomain of PARG, the macrodomain of TARG1 cannot digest the 1'-2' glycosidic bonds that make PAR chains (Sharifi et al., 2013). Instead, it hydrolyzes only the ester bond between the first ADPR and glutamic acid/aspartic acid residue, and thus removes only MARylation, not PARylation (Fig. 1). Similarly, MacroD1 and MacroD2 also favor removal of MARylation over PARylation (Fig. 1) (Rack et al., 2016; Feijs et al., 2020). However, similar to PARG, all these enzymes play important roles in DNA damage repair. It has been reported that TARG1 is required for SSB repair by redeploying X-ray repair cross-complementing group 1 (XRCC1) from one DNA damage site to another (Wei and Yu, 2016; Bütepage et al., 2018; Kassab et al., 2020). MacroD2 is also known to be recruited to DNA lesions, and participates in DNA damage repair (Golia et al., 2017). When DNA damage occurs, PARPs generate PARylation at DNA lesions. PARG is recruited to DNA lesions for dePARylation, but leaves MARylation at DNA lesions. Thus, TARG1, MacroD1, and MacroD2 may act downstream of PARG for removal of the remaining MARylation at sites of DNA damage (Bütepage et al., 2015; Feijs et al., 2020). Collectively, these macrodomains are key enzymatic domains in human ADP-ribosylhydrolases, and may act together to orchestrate deADP-ribosylation during DNA damage repair.

\section{Enzymatically inactive macrodomain}

Besides these ADP-ribosylhydrolases, other proteins such as MacroH2A and a set of PARPs also have macrodomains (Amé et al., 2004; Kustatscher et al., 2005; Yu et al., 2005; Egloff et al., 2006; Kleine et al., 2008; Poltronieri and Miwa, 2016). These macrodomains are not able to reverse ADP-ribosylation because they lack the key catalytic residues attacking the glycosidic bonds or ester bonds of ADP-ribosylation (Rack et al., 2016). However, because of their conserved secondary structure, some of these macrodomains can still bind to ADPR as "readers." A typical example is $\mathrm{MacroH} 2 \mathrm{~A}$, a histone $\mathrm{H} 2 \mathrm{~A}$ variant which can replace canonical $\mathrm{H} 2 \mathrm{~A}$ and be incorporated into the nucleosome (Kustatscher et al., 2005). However, unlike canonical H2A that contains only a global domain and two terminal tails, MacroH2A species have the macrodomain at the C-terminus, which is extended towards the outside of the nucleosome, suggesting that the macrodomain may play a key role in regulating chromatin status. Human MacroH2A species are encoded by two gene copies and have three different forms: MacroH2A1.1, MacroH2A1.2, and MacroH2A2. MacroH2A1.1 and MacroH2A1.2 are encoded by a single gene and translated by different splicing forms. The primary sequences of these three macrodomains are slightly different, and only MacroH2A1.1 recognizes ADPR, whereas MacroH2A1.2 and MacroH2A2 do not bind to ADPR (Marjanović et al., 2017; Ruiz et al., 2019). MacroH2A1.1 is able to be incorporated into sites of DNA damage. It is possible that incorporation of this unique histone variant induces local chromatin remodeling for DNA damage repair.

In addition to MacroH2A, the macrodomain of amplified in liver cancer 1 (ALC1) also recognizes ADP-ribosylation (Ahel et al., 2009). ALC1 is a nuclear polypeptide with a C-terminal macrodomain and an N-terminal DNA helicase domain. Although the DNA helicase domain may not be able to unwind double-stranded DNA, it consumes adenosine triphosphate (ATP) for sliding nucleosomes on the chromatin. The interaction between the macrodomain of ALC1 and poly(ADP-ribose) not only mediates the recruitment of ALC1 to DNA lesions, but also facilitates the sliding of nucleosomes on the chromatin. The interaction induces intermolecular changes that activate the DNA helicase domain to consume ATP.

PARPs themselves also have macrodomains. To date, 17 PARP family members have been identified in human cells (Vyas et al., 2014). Among them, PARP9, PARP14, and PARP15 have tandem macrodomains at the N-terminus (Amé et al., 2004; Beneke et al., 2004; Jankevicius et al., 2013). Since one macrodomain is sufficient to bind at least one ADPR residue, these macrodomains may recognize PARylation or multiple MARylation. As they lack the key catalytic residues, these macrodomains cannot reverse 
ADP-ribosylation. The ADPR transferase domains of these three PARPs exist at the C-terminus. However, only PARP14 and PARP15 have potent ADPR transferase activity. PARP9 has been reported to be a catalytically active ADPR transferase when it forms a complex with deltex 3-like (DTX3L) (Yang et al., 2017). It is still unclear if PARylation or multiple MARylation may promote the ADPR transferase activity of these PARPs.

\section{Structure of the macrodomain}

The crystal structures of these macrodomains of ADP-ribosylhydrolases have been solved. They share a common conformational fold, exhibiting a three-layered $\alpha-\beta-\alpha$ sandwich with a central $\beta$ sheet containing a mixture of anti-parallel and parallel strands (Rack et al., 2020). The ADPR-binding pocket in these macrodomains has an L-shaped fold, and the ADPR molecule adopts a bent conformation with the adenosine moiety located on the short side and the pyrophosphate moiety and the distal ribose located on the long side. However, the bending degree may be slightly different among these macrodomains.

The structure of the macrodomain of PARG exhibits a twisted 10 -stranded $\beta$-sheet core surrounded by two additional $\alpha$-helical subdomains on each side
(Fig. 2a) (Hassler et al., 2011; Tucker et al., 2012; Lambrecht et al., 2015). The ADPR-binding cleft is surrounded by the conserved ADPR diphosphatebinding loop and the PARG-specific GGG-X6-8-QEE motif (N744-C756), which places the catalytic residues including Gln754, Glu755, and Glu756 at the edge of the catalytic site. A third conserved Y792TGYA796 motif, termed the "Tyr-clasp," forms the boundary of the ADPR-binding cleft (Tucker et al., 2012). Glu756 works together with the catalytic water molecule to attack the glycosidic bond in PAR. The side chain of Tyr795 may be involved in substrate release. In addition, the crystal structure of PAR fragments bound to inactive Tetrahymena thermophila PARG (TTPARG) reveals that canonical PARGs, including human PARG, can predominantly act as exo-glycohydrolases owing to the high-affinity binding of the PAR terminus in exo-mode. The inherent binding preference is closely related to the conserved adenosine stacking residue Phe902 (the corresponding residue in T. thermophila PARG is Phe398) (Barkauskaite et al., 2013). Moreover, other pockets are observed in this catalytic domain, such as those on the surface of the $\mathrm{N}$-terminal extension and a deep hydrophilic pocket located on the opposite face of the catalytic center. It is possible that these pockets provide contact sites for interactions with functional partners or protein substrates of PARG (Tucker et al., 2012).
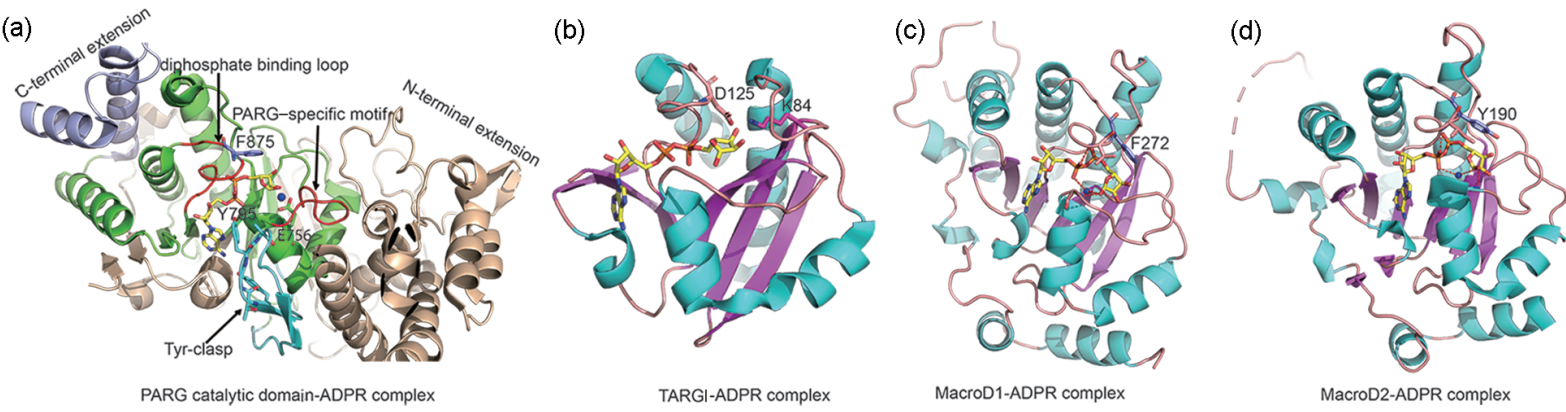

Fig. 2 Structural analysis of human adenosine diphosphate (ADP)-ribosylhydrolases. The ADP-ribose (ADPR) molecule is in yellow stick, and the catalytic water is shown in blue sphere. (a) The poly(ADP-ribose) glycohydrolase (PARG) catalytic domain is a macrodomain (Protein Data Bank (PDB) code: 4B1H). The N-terminal extension is shown in wheat cartoon, the terminal extension in light-blue, and the macrodomain core in green. The catalytic residue Glu756 is in green stick. The substrate release-related residue Tyr795 is in cyan stick, and the conserved Phe875 is in light-blue stick. The Tyr-clasp is shown in cyan. Both the ADPR diphosphate-binding loop and the PARG-specific motif are in red. (b) Structural analysis of the terminal ADP-ribose protein glycohydrolase 1 (TARG1)-ADPR complex (PDB code: 4J5S and 2L8R). ADPR is located at the surface cleft in TARG1. The catalytic residues Asp125 and Lys84 are in sticks. (c) Structural analysis of the MacroD1-ADPR complex (PDB code: 6LH4). The conserved catalysis-related residue Phe272 is shown in light-blue stick, and the conserved hydrogen bond network is in red dash. (d) Structural analysis of MacroD2-ADPR complex (PDB code: 4IQY). The conserved catalysis-related residue Tyr190 is shown in light-blue stick, and the conserved hydrogen bond network is in red dash. 
Although the TARG1 macrodomain adopts a similar three-layered $\alpha-\beta-\alpha$ sandwich, the folding is much simpler, with a six-stranded $\beta$ sheet surrounded by $\alpha$-helices on each side. The ADPR-binding pocket is formed by four surface loops (Fig. 2b). The catalytic site consists of Ser35, Lys84, and Asp125. The Lys84/Asp125 catalytic dyad is responsible for the release of ADPR from protein substrates (Sharifi et al., 2013).

Recently, we have determined the crystal structure of the MacroD1-ADPR complex (Yang et al., 2020). The MacroD1 exhibits a canonical macrodomain fold and ADPR binds to its deep substratebinding cleft (Fig. 2c). Upon ADPR binding, significant conformational changes are observed in MacroD1. The conserved Phe272, catalytic water and ADPR $\alpha$ phosphate are essential for MacroD1-mediated DPR hydrolysis. Phe272 contributes significantly to the orientation of ADPR distal ribose (Fig. 2c). This residue is relatively conserved in a subset of macrodomain-containing ADP-ribosylhydrolases, such as PARG and MacroD2 with Phe875 and Tyr190, respectively (Figs. 2a and $2 \mathrm{~d}$ ). However, the TARG1 macrodomain does not have a phenylalanine or tyrosine at the same position. Instead, the corresponding residue at the position for the aromatic amino residue is Asp125 (Fig. 2b). Thus, the conformation of ADPR in the TARG1 macrodomain is slightly different from that in MacroD1. The catalytic water is held and oriented by a conserved hydrogen-bond network in MacroD1, which is universal in the macrodomain hydrolases (Fig. 2c). MacroD2 is the closest homolog of MacroD1, and they share a very similar structural fold, with a root-mean-square deviation (RMSD) value of $1.3 \AA$ for their corresponding $\mathrm{C} \alpha$ atoms (Fig. 2d). This results in an almost identical molecular mechanism for the hydrolysis of ADP-ribosylation (Jankevicius et al., 2013). Both MacroD1 and MacroD2 belong to a subset domain of the macrodomain, namely the MacroD-type domain.

Unlike these catalytic active macrodomains, catalytic inactive macrodomains lose the key residues in the catalytic center. A typical example is MacroH2A1.1, in which the distal ribose of ADPR adopts a more extended conformation. This is caused by the replacement of the phenylalanine with asparagine (Asn316 in MacroH2A. 1.1) (Fig. 3a). Loss of the conserved phenyl group of the phenylalanine abolishes steric hindrance for the suitable conformation of the distal ribose of ADPR in the catalytic site. Although the overall structures of MacroH2A1.2 and MacroH2A2 are very similar to that of MacroH2A1.1, both MacroH2A1.2 and MacroH2A2 cannot bind to ADPR. Compared to MacroH2A1.1, three structural changes in MacroH2A1.2, including the insertion of three residues (Glu-Ile-Ser) into the adenine region, the vertical flip action of the adenine stacking residue phenylalanine, and the replacement of two Gly223-Gly224 residues by Lys224-Asp225, abolish the interaction with ADPR (Fig. 3b) (Kustatscher et al., 2005). For MacroH2A2, there structural differences render the interaction with ADPR, including: (1) a proline replacement distorts the ADPR phosphate-binding loop (Pro315 in MacroH2A2); (2) a three-amino-acid insertion occurs in the adenine-binding cleft, abolishing the interaction between Asp203 and the adenosine moiety; (3) the exchange of glycine (Gly224 in MacroH2A1.1 hydrogen-bonds the $1-\mathrm{OH}$ of the distal ribose) with charged residues (aspartate/glutamate) in MacroH2A2; (4) the flip action of the adenine stacking residue phenylalanine (Fig. 3c) (Kozlowski et al., 2018).

\section{Evolution of the macrodomain}

The macrodomain is an evolutionarily conserved domain and exists in lower organisms such as budding yeasts, bacteria, and viruses (Neuvonen and Ahola, 2009; Leung et al., 2018). In Saccharomyces cerevisiae, two proteins containing macrodomain have been identified, namely POA1 and YMR087W (Rack et al., 2016). Both are involved in the metabolism of ADPR 1"-phosphate (Apprlp), a byproduct of transfer RNA (tRNA) splicing in yeast (Shull et al., 2005). As phosphatases, these two macrodomains remove the phosphate moiety from Appr1p. POA1 is also able to remove ADPR from protein substrates in vitro, although it is unclear if this enzymatic activity plays any physiologically relevant role in vivo. However, YMR087W is inactive in ADPR hydrolysis, which may be attributed to the replacement of the conserved aromatic amino acid with alanine in YMR087W (Jankevicius et al., 2013).

Although the protein ADPR transferase has not yet been identified in bacteria, ADP-ribosylation of proteins does occur (Simon et al., 2014). 
(a)

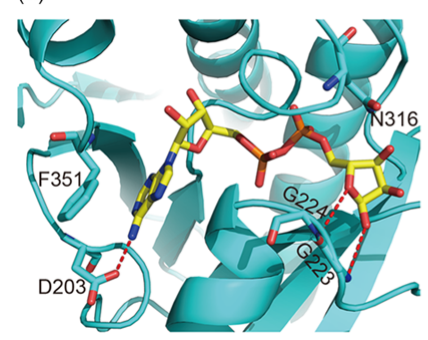

(b)

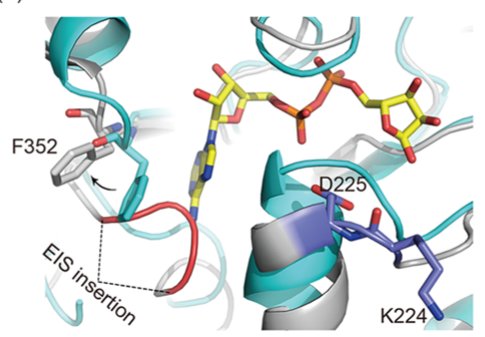

(c)

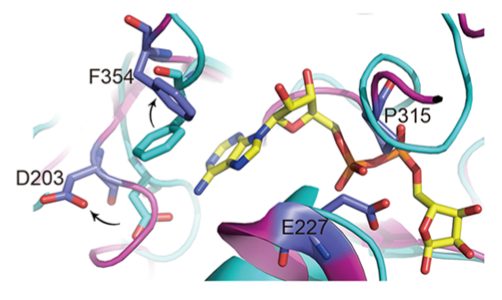

Fig. 3 Structural analysis of enzymatically inactive macrodomains including MacroH2A1.1, MacroH2A1.2, and MacroH2A2. (a) MacroH2A1.1 is able to bind adenosine diphosphate (ADP)-ribosylhydrolases (ADP)-ribose (ADPR), but is catalytically inactive for ADPR hydrolysis (Protein Data Bank (PDB) code: 3IID). MacroH2A1.1 is shown in cyan cartoon, and the adenine-binding residue Asp203, the distal ribose-interacting residues Gly223 and Gly224, and adenine-stacking residue Phe351 are in cyan sticks. The non-conserved Asn316 is also in cyan stick. The hydrogen-bond is in red dash. The ADPR is in yellow stick. (b) Structural comparison between the MacroH2A1.1-ADPR complex (PDB code: 3IID) and apo-MacroH2A1.2 (PDB code: 1ZR5). The MacroH2A1.1 and MacroH2A1.2 are in cyan and gray cartoon, respectively. The red loop indicates the insertion of three EIS residues into the adenine region. The Phe352 in MacroH2A1.2 is in gray stick and shows a vertical flip compared to the adenine stacking residue in MacroH2A1.1. Residues Lys224 and Asp225 are in light-blue sticks. (c) Structural comparison between MacroH2A1.1 and MacroH2A2 (PDB code: 6FY5). MacroH2A1.1 and MacroH2A2 are in cyan and magentas cartoon, respectively.

Macrodomains have been identified in bacterial species (Perina et al., 2014; Rack et al., 2016; Leung et al., 2018), suggesting that they may participate in ADPribosylation. In particular, Escherichia coli $\mathrm{YmdB}$ has a macrodomain protein that can hydrolyze ADPR. Other bacterial macrodomains have been identified to remove ADP-ribosylation in Staphylococcus aureus and Streptococcus pyogenes (Rack et al., 2015). Although the physiologically relevant functions of these macrodomains remain elusive, it has been reported that the macrodomain protein DarG from Mycobacterium tuberculosis acts as an antitoxin (Jankevicius et al., 2016).

\section{Macrodomains in viruses}

Macrodomains have also been identified in more than 150 types of viruses, such as hepatitis E virus, alphaviruses, and coronaviruses (Egloff et al., 2006; Malet et al., 2006, 2009; Fehr et al., 2018). The open reading frame 1 (ORF1) of the hepatitis E virus contains a macrodomain that is fused with a helicase domain and an RNA-dependent RNA polymerase (RdRp) domain (Nan et al., 2014), suggesting that the macrodomain may facilitate viral RNA replication. In alphaviruses (e.g., Chikungunya virus (CHIKV)) and coronaviruses (e.g., severe acute respiratory syndrome (SARS) and SARS coronavirus 2 (SARS-CoV-2)) (Egloff et al., 2006;
Jankevicius et al., 2013; Barkauskaite et al., 2015), the nonstructural protein $3(\mathrm{nsP} 3)$ has a macrodomain with secondary folding similar to that of human MacroD1 and MacroD2. Structural comparison between the macrodomains of CHIKV, SARS, and SARS-CoV-2 reveals the following structural characteristics: (1) The viral macrodomain exhibits a canonical macrodomain fold containing an $\alpha-\beta-\alpha$ sandwich core; (2) the central $\beta$ sheet is structurally conserved, in spite of the diverse number of $\beta$-strands in the $\beta$ sheet; (3) although the number of $\alpha$ helices located on each side of the $\beta$ sheet may vary, the positions of the common $\alpha$ helices are well conserved; (4) an aspartic acid involved in the specific binding of the adenine base is highly conserved in all macrodomains; (5) the catalytically relevant phenyl group of the key aromatic residue is conserved for ADPR hydrolysis (Fig. 4a) (Egloff et al., 2006; Malet et al., 2009; Rack et al., 2016). As a result, all these viral macrodomains, like human ADP-ribosylhydrolases, are able to reverse ADP-ribosylation. Moreover, they can dephosphorylate Appr1p, a byproduct from tRNA processing (Fehr et al., 2018). In addition, positively charged patches are observed outside of the ADPRbinding pocket (Fig. 4b), and can bind negatively charged polymers, such as PAR and RNA. Recently, it has been shown that the viral macrodomain proteins from venezuelan equine encephalitis virus (VEEV) and SARS viruses can hydrolyse ADP-ribosylation from RNA (Munnur et al., 2019). Thus, it is possible that these 

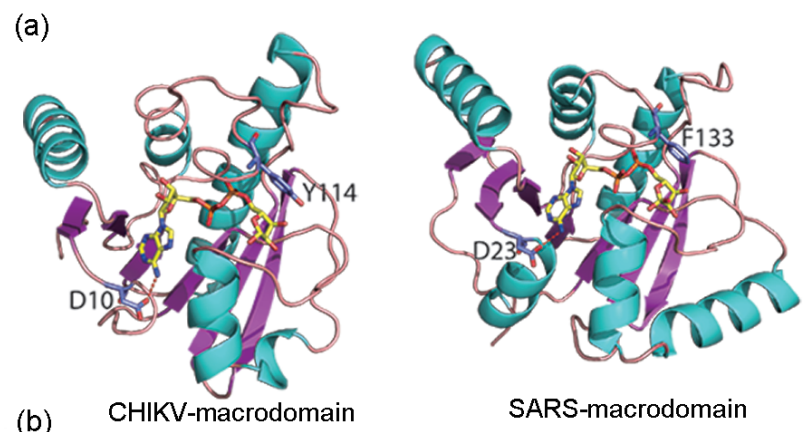

SARS-macrodomain

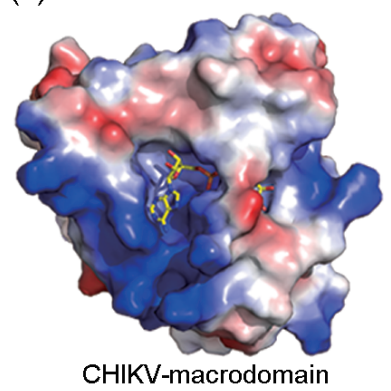

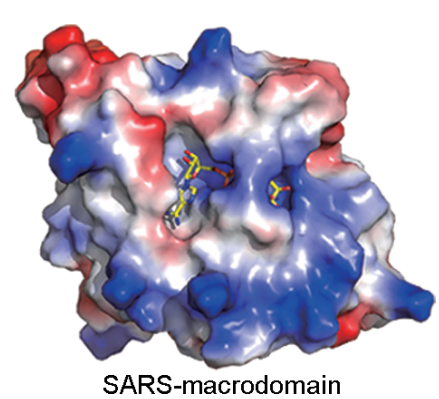

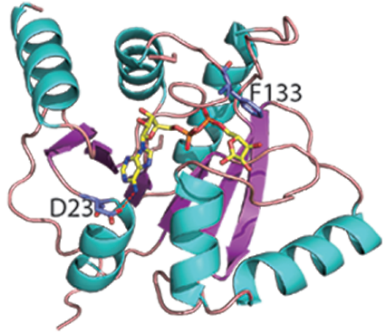

SARS-CoV-2-macrodomain

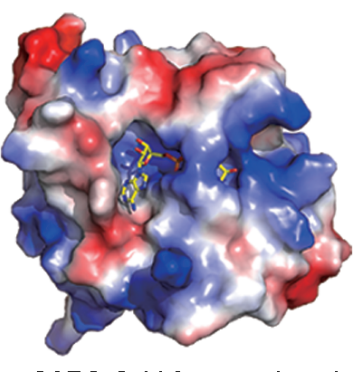

Fig. 4 Structural analysis of viral macrodomains from Chikungunya virus (CHIKV) (Protein Data Bank (PDB) code: 3GPO), severe acute respiratory syndrome (SARS) (PDB code: 2FAV), and SARS coronavirus 2 (SARS-CoV2) (PDB code: 6YWL). (a) The macrodomain structures of CHIKV, SARS, and SARS-CoV-2. The adenosine diphosphate (ADP)-ribose (ADPR) molecule is in yellow sticks. The conserved Asp and aromatic residues are shown in light-blue sticks. (b) Electrostatic surface potential maps of the macrodomains of CHIKV, SARS, and SARS-CoV-2. The positively and negatively charged patches are shown in blue and red, respectively.

viral macrodomains not only regulate RNA metabolism for their amplification, but also may render ADPribosylation and affect other cellular processes in hosts.

\section{Perspective}

Macrodomains share a similar secondary folding and tertiary structure, and have diverse biological functions ranging from DNA damage repair to viral replication (Rack et al., 2016). Due to their conserved structure, many macrodomains have ADPribosylhydrolase activity (Jankevicius et al., 2013; Rosenthal et al., 2013). Knowledge gained from the analysis of human ADP-ribosylhydrolases, may further our understanding of the biological functions and molecular mechanisms of the macrodomains during pathogen invasion (Kowieski et al., 2008; Daugherty et al., 2014), especially in relation to SARS-CoV-2. Since it is required for viral amplification (Lastarza et al., 1994; Gorbalenya et al., 2006), targeting the macrodomain could be a novel approach for suppressing virus invasion. Using in silico and biochemistry screening, small molecular inhibitors have been developed for targeting the macrodomain of PARG. These small molecular inhibitors directly block the enzymatic center of PARG, thus abolishing its catalytic activity (Chen and Yu, 2019; Houl et al., 2019; Kassab et al., 2020). Using a similar strategy, it may be possible to develop novel inhibitors that disrupt the ADP-ribosylhydrolase activity of the macrodomain of SARS-CoV-2 for the eradication of coronavirus disease 2019 (COVID-19).

\section{Acknowledgments}

The research was supported by the National Natural Science Foundation of China (No. 81874160), the Foundation of Hebei Educational Committee (No. ZD2020183), the Ministry of Education Chunhui Project, the Hebei Province Foundation for Returned Overseas Chinese Scholars (No. C20200303), and the research funds from Westlake University, Hangzhou, China.

\section{Author contributions}

Lily YU wrote the manuscript and summarized the relevant literature. Xiuhua LIU and Xiaochun YU provided the theme and design, and edited the manuscript. All authors have read and approved the final manuscript. 


\section{Compliance with ethics guidelines}

Lily YU, Xiuhua LIU, and Xiaochun YU declare that they have no conflict of interest.

This article does not contain any studies with human or animal subjects performed by any of the authors.

\section{References}

Ahel D, Hořejší Z, Wiechens N, et al., 2009. Poly(ADPribose)-dependent regulation of DNA repair by the chromatin remodeling enzyme ALC1. Science, 325(5945): $1240-1243$ https://doi.org/10.1126/science.1177321

Amé JC, Spenlehauer C, de Murcia G, 2004. The PARP superfamily. BioEssays, 26(8):882-893. https://doi.org/10.1002/bies.20085

Amé JC, Fouquerel E, Gauthier LR, et al., 2009. Radiationinduced mitotic catastrophe in PARG-deficient cells. $J$ Cell Sci, 122(Pt 12):1990-2002. https://doi.org/10.1242/jcs.039115

Audeh MW, Carmichael J, Penson RT, et al., 2010. Oral poly(ADP-ribose) polymerase inhibitor olaparib in patients with $B R C A 1$ or $B R C A 2$ mutations and recurrent ovarian cancer: a proof-of-concept trial. Lancet, 376(9737): 245-251. https://doi.org/10.1016/S0140-6736(10)60893-8

Barkauskaite E, Brassington A, Tan ES, et al., 2013. Visualization of poly(ADP-ribose) bound to PARG reveals inherent balance between exo- and endo-glycohydrolase activities. Nat Commun, 4:2164.

https://doi.org/10.1038/ncomms3164

Barkauskaite E, Jankevicius G, Ahel I, 2015. Structures and mechanisms of enzymes employed in the synthesis and degradation of PARP-dependent protein ADPribosylation. Mol Cell, 58(6):935-946. https://doi.org/10.1016/j.molcel.2015.05.007

Beneke S, Diefenbach J, Bürkle A, 2004. Poly(ADP-ribosyl)ation inhibitors: promising drug candidates for a wide variety of pathophysiologic conditions. Int J Cancer, 111(6): 813-818. https://doi.org/10.1002/ijc.20342

Bryant HE, Schultz N, Thomas HD, et al., 2005. Specific killing of BRCA2-deficient tumours with inhibitors of poly(ADP-ribose) polymerase. Nature, 434(7035):913-917. https://doi.org/10.1038/nature03443

Bütepage M, Eckei L, Verheugd P, et al., 2015. Intracellular mono-ADP-ribosylation in signaling and disease. Cells, 4(4):569-595. https://doi.org/10.3390/cells4040569

Bütepage M, Preisinger C, von Kriegsheim A, et al., 2018. Nucleolar-nucleoplasmic shuttling of TARG1 and its control by DNA damage-induced poly-ADP-ribosylation and by nucleolar transcription. Sci Rep, 8:6748. https://doi.org/10.1038/s41598-018-25137-w

Chen DW, Vollmar M, Rossi MN, et al., 2011. Identification of macrodomain proteins as novel $O$-acetyl-ADP-ribose deacetylases. J Biol Chem, 286(15):13261-13271. https://doi.org/10.1074/jbc.M110.206771
Chen SH, Yu XC, 2019. Targeting dePARylation selectively suppresses DNA repair-defective and PARP inhibitorresistant malignancies. Sci Adv, 5(4):eaav4340. https://doi.org/10.1126/sciadv.aav4340

Daugherty MD, Young JM, Kerns JA, et al., 2014. Rapid evolution of PARP genes suggests a broad role for ADPribosylation in host-virus conflicts. PLoS Genet, 10(5): e1004403. https://doi.org/10.1371/journal.pgen.1004403

Egloff MP, Malet H, Putics A, et al., 2006. Structural and functional basis for ADP-ribose and poly(ADP-ribose) binding by viral macro domains. J Virol, 80(17):8493-8502. https://doi.org/10.1128/JVI.00713-06

Fauzee NJS, Pan J, Wang YL, 2010. PARP and PARG inhibitors - new therapeutic targets in cancer treatment. Pathol Oncol Res, 16(4):469-478. https://doi.org/10.1007/s12253-010-9266-6

Fehr AR, Jankevicius G, Ahel I, et al., 2018. Viral macrodomains: unique mediators of viral replication and pathogenesis. Trends Microbiol, 26(7):598-610. https://doi.org/10.1016/j.tim.2017.11.011

Feijs KLH, Forst AH, Verheugd P, et al., 2013. Macrodomaincontaining proteins: regulating new intracellular functions of mono(ADP-ribosyl)ation. Nat Rev Mol Cell Biol, 14(7):443-451. https://doi.org/10.1038/nrm3601

Feijs KLH, Cooper CDO, Žaja R, 2020. The controversial roles of ADP-ribosyl hydrolases MACROD1, MACROD2 and TARG1 in carcinogenesis. Cancers (Basel), 12(3):604. https://doi.org/10.3390/cancers12030604

Fisher AEO, Hochegger H, Takeda S, et al., 2007. Poly(ADPribose) polymerase 1 accelerates single-strand break repair in concert with poly(ADP-ribose) glycohydrolase. Mol Cell Biol, 27(15):5597-5605. https://doi.org/10.1128/MCB.02248-06

Garufi G, Palazzo A, Paris I, et al., 2020. Neoadjuvant therapy for triple-negative breast cancer: potential predictive biomarkers of activity and efficacy of platinum chemotherapy, PARP- and immune-checkpoint-inhibitors. Expert Opin Pharmacother, 21(6):687-699. https://doi.org/10.1080/14656566.2020.1724957

Gogola E, Duarte AA, de Ruiter JR, et al., 2019. Selective loss of PARG restores PARylation and counteracts PARP inhibitor-mediated synthetic lethality. Cancer Cell, 35(6): 950-952. https://doi.org/10.1016/j.ccell.2019.05.012

Golia B, Moeller GK, Jankevicius G, et al., 2017. ATM induces MacroD2 nuclear export upon DNA damage. Nucleic Acids Res, 45(1):244-254. https://doi.org/10.1093/nar/gkw904

Gorbalenya AE, Enjuanes L, Ziebuhr J, et al., 2006. Nidovirales: evolving the largest RNA virus genome. Virus Res, 117(1):17-37. https://doi.org/10.1016/j.virusres.2006.01.017

Grundy GJ, Rulten SL, Zeng ZH, et al., 2013. APLF promotes the assembly and activity of non-homologous end joining protein complexes. EMBO J, 32(1):112-125. https://doi.org/10.1038/emboj.2012.304 
Hassler M, Jankevicius G, Ladurner AG, 2011. PARG: a macrodomain in disguise. Structure, 19(10):1351-1353. https://doi.org/10.1016/j.str.2011.09.007

Houl JH, Ye Z, Brosey CA, et al., 2019. Selective small molecule PARG inhibitor causes replication fork stalling and cancer cell death. Nat Commun, 10:5654. https://doi.org/10.1038/s41467-019-13508-4

Jankevicius G, Hassler M, Golia B, et al., 2013. A family of macrodomain proteins reverses cellular mono-ADPribosylation. Nat Struct Mol Biol, 20(4):508-514. https://doi.org/10.1038/nsmb.2523

Jankevicius G, Ariza A, Ahel M, et al., 2016. The toxinantitoxin system DarTG catalyzes reversible ADPribosylation of DNA. Mol Cell, 64(6):1109-1116. https://doi.org/10.1016/j.molcel.2016.11.014

Kassab MA, Yu XC, 2019. The role of dePARylation in DNA damage repair and cancer suppression. DNA Repair (Amst), 76:20-29. https://doi.org/10.1016/j.dnarep.2019.02.002

Kassab MA, Yu LL, Yu XC, 2020. Targeting dePARylation for cancer therapy. Cell Biosci, 10:7. https://doi.org/10.1186/s13578-020-0375-y

Kaufmann T, Grishkovskaya I, Polyansky AA, et al., 2017. A novel non-canonical PIP-box mediates PARG interaction with PCNA. Nucleic Acids Res, 45(16):9741-9759. https://doi.org/10.1093/nar/gkx604

Kleine H, Poreba E, Lesniewicz K, et al., 2008. Substrateassisted catalysis by PARP10 limits its activity to monoADP-ribosylation. Mol Cell, 32(1):57-69. https://doi.org/10.1016/j.molcel.2008.08.009

Kowieski TM, Lee S, Denu JM, 2008. Acetylation-dependent ADP-ribosylation by Trypanosoma brucei Sir2. J Biol Chem, 283(9):5317-5326. https://doi.org/10.1074/jbc.M707613200

Kozlowski M, Corujo D, Hothorn M, et al., 2018. MacroH2A histone variants limit chromatin plasticity through two distinct mechanisms. EMBO Rep, 19(10):e44445. https://doi.org/10.15252/embr.201744445

Krietsch J, Rouleau M, Pic É, et al., 2013. Reprogramming cellular events by poly(ADP-ribose)-binding proteins. Mol Aspects Med, 34(6):1066-1087. https://doi.org/10.1016/j.mam.2012.12.005

Kustatscher G, Hothorn M, Pugieux C, et al., 2005. Splicing regulates NAD metabolite binding to histone macroH2A. Nat Struct Mol Biol, 12(7):624-625. https://doi.org/10.1038/nsmb956

Lambrecht MJ, Brichacek M, Barkauskaite E, et al., 2015. Synthesis of dimeric ADP-ribose and its structure with human poly(ADP-ribose) glycohydrolase. $\mathrm{J} \mathrm{Am} \mathrm{Chem}$ Soc, 137(10):3558-3564. https://doi.org/10.1021/ja512528p

LaStarza MW, Lemm JA, Rice CM, 1994. Genetic analysis of the nsP3 region of sindbis virus: evidence for roles in minus-strand and subgenomic RNA synthesis. J Virol, 68(9):5781-5791. https://doi.org/10.1128/JVI.68.9.5781-5791.1994

Leung AKL, McPherson RL, Griffin DE, 2018. Macrodomain ADP-ribosylhydrolase and the pathogenesis of infec- tious diseases. PLoS Pathog, 14(3):e1006864. https://doi.org/10.1371/journal.ppat.1006864

Li M, Yu X, 2015. The role of poly(ADP-ribosyl)ation in DNA damage response and cancer chemotherapy. Oncogene, 34(26):3349-3356. https://doi.org/10.1038/onc.2014.295

Liu C, Vyas A, Kassab MA, et al., 2017. The role of poly ADP-ribosylation in the first wave of DNA damage response. Nucleic Acids Res, 45(14):8129-8141. https://doi.org/10.1093/nar/gkx565

Malet H, Dalle K, Brémond N, et al., 2006. Expression, purification and crystallization of the SARS-CoV macro domain. Acta Cryst Sect F Struct Biol Cryst Commun, 62(Pt 4):405-408. https://doi.org/10.1107/S1744309106009274

Malet H, Coutard B, Jamal S, et al., 2009. The crystal structures of Chikungunya and Venezuelan equine encephalitis virus nsP3 macro domains define a conserved adenosine binding pocket. $J$ Virol, 83(13):6534-6545. https://doi.org/10.1128/JVI.00189-09

Marjanović MP, Hurtado-Bagès S, Lassi M, et al., 2017. MacroH2A1.1 regulates mitochondrial respiration by limiting nuclear $\mathrm{NAD}^{+}$consumption. Nat Struct Mol Biol, 24(11):902-910. https://doi.org/10.1038/nsmb.3481

Michels J, Vitale I, Saparbaev M, et al., 2014. Predictive biomarkers for cancer therapy with PARP inhibitors. Oncogene, 33(30):3894-3907. https://doi.org/10.1038/onc.2013.352

Min W, Cortes U, Herceg Z, et al., 2010. Deletion of the nuclear isoform of poly(ADP-ribose) glycohydrolase (PARG) reveals its function in DNA repair, genomic stability and tumorigenesis. Carcinogenesis, 31(12):2058-2065. https://doi.org/10.1093/carcin/bgq205

Munnur D, Ahel I, 2017. Reversible mono-ADP-ribosylation of DNA breaks. FEBS J, 284(23):4002-4016. https://doi.org/10.1111/febs.14297

Munnur D, Bartlett E, Mikolčević P, et al., 2019. Reversible ADP-ribosylation of RNA. Nucleic Acids Res, 47(11): 5658-5669. https://doi.org/10.1093/nar/gkz305

Nan YC, Yu Y, Ma ZX, et al., 2014. Hepatitis E virus inhibits type I interferon induction by ORF1 products. $J$ Virol, 88(20):11924-11932. https://doi.org/10.1128/JVI.01935-14

Neuvonen M, Ahola T, 2009. Differential activities of cellular and viral macro domain proteins in binding of ADPribose metabolites. $J$ Mol Biol, 385(1):212-225. https://doi.org/10.1016/j.jmb.2008.10.045

Patel CN, Koh DW, Jacobson MK, et al., 2005. Identification of three critical acidic residues of poly(ADP-ribose) glycohydrolase involved in catalysis: determining the PARG catalytic domain. Biochem J, 388(Pt 2):493-500. https://doi.org/10.1042/BJ20040942

Perina D, Mikoč A, Ahel J, et al., 2014. Distribution of protein poly(ADP-ribosyl)ation systems across all domains of life. DNA Repair (Amst), 23:4-16. https://doi.org/10.1016/j.dnarep.2014.05.003 
Pillay N, Tighe A, Nelson L, et al., 2019. DNA replication vulnerabilities render ovarian cancer cells sensitive to poly(ADP-ribose) glycohydrolase inhibitors. Cancer Cell, 35(3):519-533.e8. https://doi.org/10.1016/j.ccell.2019.02.004

Poltronieri P, Miwa M, 2016. Editorial (thematic issue: overview on ADP ribosylation and PARP superfamily of proteins). Curr Protein Pept Sci, 17(7):630-632. https://doi.org/10.2174/138920371707160908172601

Powell SN, Kachnic LA, 2003. Roles of BRCA1 and BRCA2 in homologous recombination, DNA replication fidelity and the cellular response to ionizing radiation. Oncogene, 22(37):5784-5791 https://doi.org/10.1038/sj.onc. 1206678

Rack JGM, Morra R, Barkauskaite E, et al., 2015. Identification of a class of protein ADP-ribosylating sirtuins in microbial pathogens. Mol Cell, 59(2):309-320. https://doi.org/10.1016/j.molcel.2015.06.013

Rack JGM, Perina D, Ahel I, 2016. Macrodomains: structure, function, evolution, and catalytic activities. Annu Rev Biochem, 85:431-454. https://doi.org/10.1146/annurev-biochem-060815-014935

Rack JGM, Palazzo L, Ahel I, 2020. (ADP-ribosyl)hydrolases: structure, function, and biology. Genes Dev, 34(5-6):263-284 . https://doi.org/10.1101/gad.334631.119

Rosenthal F, Feijs KLH, Frugier E, et al., 2013. Macrodomaincontaining proteins are new mono-ADP-ribosylhydrolases. Nat Struct Mol Biol, 20(4):502-507. https://doi.org/10.1038/nsmb.2521

Ruiz PD, Hamilton GA, Park JW, et al., 2019. MacroH2A1 regulation of poly(ADP-ribose) synthesis and stability prevents necrosis and promotes DNA repair. Mol Cell Biol, 40(1):e00230-19. https://doi.org/10.1128/MCB.00230-19

Sharifi R, Morra R, Appel CD, et al., 2013. Deficiency of terminal ADP-ribose protein glycohydrolase TARG1/ C6orf130 in neurodegenerative disease. EMBO J, 32(9): 1225-1237. https://doi.org/10.1038/emboj.2013.51

Shirai H, Poetsch AR, Gunji A, et al., 2013. PARG dysfunction enhances DNA double strand break formation in Sphase after alkylation DNA damage and augments different cell death pathways. Cell Death Dis, 4:e656. https://doi.org/10.1038/cddis.2013.133
Shull NP, Spinelli SL, Phizicky EM, 2005. A highly specific phosphatase that acts on ADP-ribose 1"-phosphate, a metabolite of tRNA splicing in Saccharomyces cerevisiae. Nucleic Acids Res, 33(2):650-660. https://doi.org/10.1093/nar/gki211

Simon NC, Aktories K, Barbieri JT, 2014. Novel bacterial ADP-ribosylating toxins: structure and function. Nat Rev Microbiol, 12(9):599-611. https://doi.org/10.1038/nrmicro3310

Slade D, 2020. PARP and PARG inhibitors in cancer treatment. Genes Dev, 34(5-6):360-394. https://doi.org/10.1101/gad.334516.119

Talhaoui I, Lebedeva NA, Zarkovic G, et al., 2016. Poly(ADPribose) polymerases covalently modify strand break termini in DNA fragments in vitro. Nucleic Acids Res, 44(19): 9279-9295. https://doi.org/10.1093/nar/gkw675

Tucker JA, Bennett N, Brassington C, et al., 2012. Structures of the human poly(ADP-ribose) glycohydrolase catalytic domain confirm catalytic mechanism and explain inhibition by ADP-HPD derivatives. PLoS ONE, 7(12): e50889. https://doi.org/10.1371/journal.pone.0050889

Vyas S, Matic I, Uchima L, et al., 2014. Family-wide analysis of poly(ADP-ribose) polymerase activity. Nat Commun, $5: 4426$. https://doi.org/10.1038/ncomms5426

Wei HT, Yu XC, 2016. Functions of PARylation in DNA damage repair pathways. Genomics Proteomics Bioinformatics, 14(3):131-139. https://doi.org/10.1016/j.gpb.2016.05.001

Yang CS, Jividen K, Spencer A, et al., 2017. Ubiquitin modification by the E3 ligase/ADP-ribosyltransferase Dtx3L/ Parp9. Mol Cell, 66(4):503-516.e5. https://doi.org/10.1016/j.molcel.2017.04.028

Yang XY, Ma YL, Li YM, et al., 2020. Molecular basis for the MacroD1-mediated hydrolysis of ADP-ribosylation. DNA Repair (Amst), 94:102899. https://doi.org/10.1016/j.dnarep.2020.102899

Yu M, Schreek S, Cerni C, et al., 2005. PARP-10, a novel Myc-interacting protein with poly(ADP-ribose) polymerase activity, inhibits transformation. Oncogene, 24(12): 1982-1993.

https://doi.org/10.1038/sj.onc.1208410 